\title{
EG-Sozialpolitik nach Verflechtungsfalle und Entscheidungslücke: Bewertungsmaßstäbe und Entwicklungstrends
}

\author{
Gerda Falkner
}

Bis in die 1990er Jahre befand sich die EG-Sozialpolitik infolge weitestgehender Einstimmigkeitserfordernisse in einer, Politikverflechtungsfalle' (Fritz W. Scharpf), die noch durch eine, korporatistische Entscheidungslücke' (Wolfgang Streeck) ergänzt wurde. Der Maastrichter Vertrag brachte allerdings signifikante Neuerungen in Hinblick auf Handlungskompetenzen, Mehrheitsvoten und die korporatistische Einbeziehung der Sozialpartnerverbände. Dass diese Potenziale entgegen vielfachen Erwartungen auch praktisch zur Anwendung kamen, heißt allerdings noch nicht, dass die Problemlösungskapazität der EG-Sozialpolitik schon zufrieden stellend wäre. Dieser Beitrag analysiert unterschiedliche Beurteilungsmaßstäbe dafür und zeigt auf, dass die ,soziale Dimension' bei Anwendung von inhaltlich vergleichsweise eng definierten Ansprüchen mittlerweile besser abschneidet, als oft erwartet wurde (Schließung akuter arbeitsrechtlicher Lücken; Kommissionsvorschläge im Vergleich zu Ratsbeschlïssen). Noch tut die Union allerdings wenig, um marktinduziertem Druck auf soziale Standards in den Mitgliedstaaten entgegenzuwirken. Bislang kaum ernsthaft diskutiert wurde auch die Nützung jener Potenziale für sozialgestaltende Politik aufsupranationaler Ebene, die heute angesichts offener Märkte in den Mitgliedstaaten immer weniger bestehen. Als Entwicklungstrend zeichnet sich in allerjüngster Zeit eine völlig neue Rolle für die EG-Sozialpolitik im 21. Jahrhundert ab: als Motor und zugleich Korsett für auf nationaler Ebene entschiedene Reformen.

\section{Einleitung*}

Die Beurteilung der ,sozialen Dimension' der europäischen Integration wurde berechtigtermaßen mit der Einschätzung von zum Teil gefüllten Gläsern als halb voll bzw. halb leer verglichen (vgl. Ross 1994; Streeck 1994; Goetschy 1994). Neben der Koexistenz von eher optimistischen mit pessimistischen Gesamtkonklusionen sticht jedoch bei genauerer Betrachtung der Fülle an einschlägiger Literatur auch ins Auge, dass die Bewertungsmaßstäbe oft eher implizite sind denn detailliert ausgeführte und systematisch begründete. Der vorliegende Beitrag stellt demgegenüber gerade die Diskussion unterschiedlicher Indikatoren für die Problemlösungsfähigkeit der EG im Sozialbereich ins Zentrum und versucht eine Einschätzung vor jedem dieser Maßstäbe.

\footnotetext{
* Für Anregungen danke ich den beiden anonoymen Gutachtern der PVS, dem Graduiertenkolleg „Die Zukunft des europäischen Sozialstaats “ an der Universität Göttingen, und den Teilnehmern der Konferenz „Wie problemlösungsfähig ist die EU?“ der DGPW am 29. - 31. Oktober 1998, wo Teile dieses Beitrags in einer Frühfassung diskutiert wurden.
} 
Dabei soll in einem ersten Schritt die Entscheidungsfähigkeitder EG als eine grundlegende Komponente der Problemlösungsfähigkeit diskutiert werden. Einen wichtigen Stellenwert nehmen dabei die Maßstäbe der „Politikverflechtungsfalle“ und der „Entscheidungslücke“ ein, an denen die EG-Sozialpolitik in der Vergangenheit gemessen und mit guten Gründen als mangelhaft bezeichnet wurde. Die in den 1990er Jahren vollzogene Reform der sozialen Dimension hat jedoch die rechtliche wie auch die praktisch-politische Kapazität der EG zur Verabschiedung sozialrechtlicher Akte, auch auf Grundlage korporatistischer Verfahren, maßgeblich vergrößert. Dies stellt die Sozialwissenschaften vor die Herausforderung, noch spezifischere Beurteilungskriterien für die Problemlösungsfähigkeit der EG zu entwickeln, um den erreichten Fortschritten einerseits Rechnung zu tragen, sie aber andererseits nicht voreilig überzubewerten.

Dieser Beitrag umreißt in einem ersten Schritt Grundzüge und Entwicklung der EG-Sozialpolitik bis Anfang der 1990er Jahre (2). Sie wurde treffend durch die politikwissenschaftlichen Ansätze der Politikverflechtungsfalle und der Entscheidungslücke charakterisiert, die verschiedene Facetten der Entscheidungs(un)fähigkeit in den Mittelpunkt stellen (3). Erst der Maastrichter Vertrag brachte signifikante Neuerungen auf dieser Ebene (4). In der Folge werden vier verschiedene Kriterien zur Bewertung der aktuellen sozialpolitischen Problemlösungskapazität der EU diskutiert, die zu jeweils unterschiedlichen Ergebnissen führen (5). Abschließend wird der jüngste Trend im Rahmen der sozialen Dimension der europäischen Integration analysiert: Seit den späten 1990er Jahren wird der EU in zunehmendem Maße eine neue Rolle als Motor und Korsett für nationale Reformen zugewiesen (6).

\section{EG-Sozialpolitik vor Maastricht}

Die Urfassung des Vertrages zur Gründung der Europäischen Wirtschaftsgemeinschaft ${ }^{1}$ aus 1957 enthielt zwar einen Titel III „Die Sozialpolitik“. Neben Bestimmungen zum Europäischen Sozialfonds ${ }^{2}$ enthielt er jedoch trotz der Überschrift „Sozialvorschriften“ eher feierliche Erklärungen als konkrete Handlungsaufträge. Jahrzehntelang besaß die $\mathrm{EG}^{3}$ also kaum explizite Handlungsermächtigungen im sozialpolitischen Bereich.

Die letztlich unverbindlichen Kompromissformulierungen resultierten aus einem grundlegenden Richtungsstreit, der in den Verhandlungen über die Römischen Verträge zwischen neo-liberaler und sozial-interventionistischer Schule ausgetragen worden war. Letztere hatte (zumindest kurzfristig erfolglos) argumentiert, dass zur Herstellung gerechter

1 Der Maastrichter Vertrag 1992 benannte diese in „Europäische Gemeinschaft“ um, der entsprechende Vertrag ist daher nunmehr der „EG-Vertrag“ oder EGV.

2 Dieser stellt entgegen seinem weiter gefassten Namen ein rein arbeitsmarktpolitisches Instrument zur Kofinanzierung von Umsiedelungen und Umschulungen von Arbeitskräften dar.

3 Auch die Verträge zur Errichtung der Europäischen Gemeinschaft für Kohle und Stahl (1951) und der Europäischen Atomgemeinschaft (1957) enthielten einige wenige Bestimmungen mit unmittelbar sozialpolitischer Bedeutung (z.B. Freizügigkeit für Arbeitskräfte im Montanbereich und spezielle Hilfen etwa im Falle von Umsiedelung oder Umschulung; Schutz der Arbeitskräfte in Atomkraftwerken). Sie sind jedoch wegen des engen sektorspezifischen Anwendungsbereichs im Unterschied zum E(W)GVertrag, der ja eine sektorübergreifende ökonomische Integration vorsieht, für die allgemeine Fragestellung dieses Beitrags weniger relevant. 
Wettbewerbsbedingungen für die Unternehmen aller EWG-Länder auch ein gewisser Grad an sozialpolitischer Harmonisierung notwendig sei. Die Gegenseite betrachtete demgegenüber Sozialkosten als nur eine unter vielen Wettbewerbsbedingungen und nicht als „,künstliche Kosten“. Mit dem Argument, das unbehinderte Spiel der Marktkräfte bringe jedenfalls die effizienteste Allokation des Kapitals und im Endergebnis einen Wohlstandsgewinn für alle Beteiligten, wandte sich so v.a. die deutsche Delegation gegen sozialpolitische Eingriffe in den freien Wettbewerb auf EG-Ebene (vgl. z.B. Beutler et al. 1987: 437; Gerbet 1983: 221).

Letztendlich setzte sich 1957 ein Kompromiss mit Übergewicht zu Gunsten der sogenannten „Nicht-Kosten-Doktrin“ (Walter Hallstein zitiert in Nelhans 1975: 138), also der Argumentationslinie der Neoliberalen, durch. Es wurde kein eigenes Verfahren zur sozialpolitischen Rechtsetzung der Gemeinschaft eingerichtet, sondern weitgehend auf einen Automatismus der „Angleichung auf dem Wege des Fortschritts“ gesetzt. Die so genannten Sozialvorschriften im EWGV enthielten daher keine konkreten Handlungsermächtigungen und auch nur punktuelle Regelungen mit materiellem sozialpolitischen Gehalt (vgl. Pipkorn 1986, IA 56: 6), etwa über die Gleichbehandlung der Geschlechter durch die Mitgliedstaaten. Im Unterschied zum Agrar-, Wettbewerbs- oder Verkehrssektor verfügte der EWGV damit im Sozialbereich über keinen allgemeinen Auftrag zu regulativer Intervention, etwa zur Angleichung des Arbeitsrechts oder zur Vereinheitlichung der Systeme der sozialen Sicherheit (z.B. Däubler 1989c: 82). ${ }^{4}$ Sozialpolitik sollte vielmehr nach dem Wortlaut des Vertrages nur in wenigen Teilaspekten Gegenstand von EG-Aktivitäten sein. Verordnungen oder Richtlinien mit direkt sozialpolitischem Bezug wurden 1957 neben dem Tätigkeitsbereich des Sozialfonds explizit nur zur sozialen Absicherung der Freizügigkeit von Arbeitskräften vorgesehen (Artikel 51 EWGV, jetzt Artikel 42 EGV). ${ }^{5}$ Lediglich auf dem Umweg über die so genannten subsidiären Kompetenznormen des EWGV konnten darüber hinaus Beschlüsse zur Angleichung sozialrechtlicher Regelungen in den Mitgliedstaaten gefasst werden. ${ }^{6}$ Diese „Hintertür “ für sozialpolitische EG-Intervention verlangt allerdings Einstimmigkeit unter den Ratsmitgliedern. Ihre Nutzung blieb aus diesem Grunde auch auf Einzelfälle beschränkt.

4 Dementsprechend wurden der EG-Kommission im sozialen Bereich nur sehr eingeschränkte Befugnisse übertragen: Gemäß Artikel 118 EWGV (jetzt Artikel 137 EGV) soll sie durch Untersuchungen, Stellungnahmen und die Vorbereitung von Beratungen - also jedenfalls für die Mitgliedstaaten nicht verbindlich - tätig werden. Eine Vorlage von Vorschlägen zum Erlass verbindlicher Rechtsakte wie auf vielen anderen Gebieten war hier nicht vorgesehen. Damit wurde die Zuständigkeit der Mitgliedstaaten (und nicht der Gemeinschaft) auf sozialpolitischem Gebiet bestätigt.

5 Die Herstellung der Freizügigkeit für ArbeitnehmerInnen sollte auch von einem System zur Sicherstellung erworbener sozialversicherungsrechtlicher Ansprüche begleitet werden. Diese EG-Kompetenz fügte sich nahtlos in den ökonomischen Rahmen der Errichtung des Gemeinsamen Marktes ein, da dieser ja auch die freie Bewegung des Produktionsfaktors Arbeit umfassen sollte. Zugleich wurde bei EWG-Vertragsabschluss darin aber auch eine beschäftigungspolitische Maßnahme gesehen, da davon ausgegangen wurde, dass Arbeitskräfte von Krisengebieten in solche mit Vollbeschäftigung auswandern würden (Däubler 1989c: 81 m.w.N.).

6 Artikel 235 EWGV (jetzt Artikel 308 EGV) erlaubt ein Tätigwerden der Gemeinschaft zur Verwirklichung eines (sei es auch allgemeinen) „Vertragsziels“ in jene Fällen, wo entsprechende Befugnisse nicht ausdrücklich vorgesehen sind. Artikel 100 (jetzt Artikel 94 EGV) erlaubt die Angleichung von Rechtsund Verwaltungsvorschriften, die sich unmittelbar auf den Gemeinsamen Markt auswirken. 
Nachdem sich die EWG-Integration anfangs auf die Einführung der EG-weiten Freizügigkeit für Arbeitskräfte und die Tätigkeit des Sozialfonds beschränkt hatte, ${ }^{7}$ begann 1972 eine Phase aktiverer Sozialpolitik. Auf ihrer Pariser Gipfelkonferenz hielten die Staats- und Regierungsoberhäupter der Mitgliedstaaten fest, dass wirtschaftliche Expansion kein Selbstzweck sein solle, sondern dazu dienen müsse, die Lebensqualität und den Lebensstandard der Bevölkerung zu heben. Sie gaben die Ausarbeitung eines sozialpolitischen Aktionsprogramms in Auftrag. In diesem Akt (ABl. 74/C 13/1) anerkannte der Rat 1974 explizit, dass für die Verwirklichung der darin angeführten drei Hauptziele (Bekämpfung der Arbeitslosigkeit, Verbesserung der Lebens- und Arbeitsbedingungen sowie weiter gehende Beteiligung der Sozialpartner) Aktivitäten der EG erforderlich wären und dass zu ihrer Verwirklichung auch Artikel 235 EWGV (jetzt Artikel 308 EGV) als Rechtsgrundlage herangezogen werden könne. In der folgenden Phase bis etwa 1980 wurden mehrere relevante Richtlinien erlassen und auf die Artikel 100 und/oder 235 EWGV gestützt (jetzt Artikel 94 und 308 EGV). Sie enthielten arbeitsrechtliche Regelungen (Wahrung von bestimmten Arbeitnehmerrechten in Fällen von Betriebsübergang, Massenentlassung oder Insolvenz des Arbeitgebers) und schrieben teils die Gleichbehandlung der Geschlechter im Arbeitsleben vor (z.B. in den Bereichen soziale Sicherheit, selbstständige Erwerbstätigkeit, Arbeitsbedingungen).

Schon bald nach Verabschiedung des Aktionsprogramms folgten jedoch ein Einbruch in der internationalen Wirtschaftsentwicklung sowie eine Änderung der nationalen Wirtschafts- und Sozialpolitiken vieler EG-Länder hin zum Neoliberalismus. Viele Vorschläge des Aktionsprogramms aus 1974 wurden blockiert. Institutionelle Rahmenbedingung dafür war die Vetomöglichkeit der Ratsdelegationen. Das in der zweiten Hälfte der 80er Jahre die EG-Politik prägende Binnenmarktprogramm hatte aus diesem Grund auch vorerst keine „soziale Dimension“ ${ }^{8}$ Ohne diesen politischen Preis wäre die erforderliche Einstimmigkeit im Ministerrat nicht zu Stande gekommen: „Hätten wir die sozialen Fragen mit den wirtschaftspolitischen Regelungen verknüpft, käme keines der beiden Vorhaben von der Stelle“ (Kommissar Cockfield, zitiert bei Fels 1989: Fn. 1). Als die Kommission zumindest ein Aktionsprogramm zur teilweisen Umsetzung der Sozialcharta aus 1989 ausarbeitete, wurden die vorgeschlagenen 48 Gemeinschaftsmaßnahmen ${ }^{9}$ im Rat nur so schleppend bearbeitet, dass die „soziale Dimension der EG“ Ende der 1980er und Anfang der 90er Jahre völlig blockiert schien (z.B. Pochet 1991: 4f.; Vogel-Polsky 1989 und 1990). ${ }^{10}$

7 Dieser teilfinanzierte schon bis 1970 die Umsiedlung oder Umschulung von ca. 800.000 Arbeitslosen (vgl. Beretta-Bericht 1987: 25).

8 Trotz vehementer Proteste des EP, mancher Mitgliedstaaten und der Arbeitnehmervertretung auf EG-Ebene (vgl. etwa Knigge 1989: 18; Entschließung des EP zur Verzögerung bei der Beschlussfassung zur Stärkung des Wirtschaftlichen und Sozialen Zusammenhalts in der Gemeinschaft und zum Verhältnis dieser Option und der Verwirklichung des Binnenmarktes, ABl. 88/C 49/118).

9 Davon waren 20 legislativen Charakters und wiederum zehn davon im technischen Arbeitsschutz.

10 Im Oktober 1991 drückte sogar der Präsident des Europäischen Parlaments, Enrique Baron, öffentlich seine Bedenken über die paralysierende Blockadesituation im Sozialbereich aus (Agence Europe 14 Oktober 1991: 9). Vielfach wurde verlangt, die in Hinblick auf die Errichtung einer Wirtschafts- und Währungsunion ins Auge gefasste Novellierung des EG-Vertragsgebäudes solle nicht zuletzt auch neue Kompetenzgrundlagen und Entscheidungsmuster für die Sozialpolitik bringen (vgl. z.B. Schulz 1991; Schulte 1991). 


\section{Politikverflechtungsfalle und Entscheidungslücke}

Die EG-Sozialpolitik unterlag damit den von Scharpf (1985) analysierten Bedingungen der „Politikverflechtungsfalle“. ${ }^{11}$ Jene Strukturmerkmale der EG, die für die „scheinbar paradoxe Koexistenz von Frustration und Stabilität und von quantitativer Erweiterung und qualitativer Stagnation“ europäischer Politik verantwortlich waren, glichen in ihren wesentlichen Eigenschaften den Funktionsbedingungen des deutschen Föderalismus, die unter dem Begriff der „Politikverflechtung“ "beschrieben worden waren (Scharpf 1985: 324). Dies sind erstens die Abhängigkeit der Entscheidungen auf höherer Ebene von der Zustimmung von Regierungen der unteren Entscheidungsebene (deren institutionelles Eigeninteresse nicht durch ein Repräsentationsprinzip gefiltert wird), und zweitens die Tatsache, dass diese $\mathrm{Zu}$ stimmung einstimmigerteilt werden muss (Scharpf 1985: 334). ${ }^{12}$ Scharpf ging dabei von der Arbeitshypothese aus, dass „die institutionellen Strukturen der EG suboptimale Politikergebnisse systematisch begünstigen“ (Scharpf 1985: 324), vor allem weil ein problemlösungsorientiertes Verhandeln gegenüber dem in der Politikverflechtung typischen bargaining sehr schlechte Bedingungen vorfinde.

Wie oben erläutert waren in der EG-Sozialpolitik vor Maastricht die typischen Bedingungen einer Politikverflechtungsfalle gegeben: die ihre nationalen Regierungen vertretenden Minister im Rat mussten EG-Sozialrecht fast ohne Ausnahme einstimmig entscheiden.

11 Mit der einzigen Ausnahme von Artikel 118a EWGV (jetzt Art. 138 EGV), der ab 1987 die Verabschiedung von Mindeststandards im Bereich von Sicherheit und Gesundheitsschutz am Arbeitsplatz mit qualifizierter Mehrheit im Rat erlaubte (vgl. dazu unten).

12 Im Original spricht Scharpf davon, dass die Zustimmung „einstimmig oder fast einstimmig “ (Scharpf 1985: 334) sein muss. Dementsprechend stellt sich in Hinblick auf die EG die Frage, ob qualifizierte Mehrheitsvoten im Ministerrat noch immer in die Kategorie „fast einstimmig“ fallen könnten. Gegen eine solche Einordnung sprechen im Text Scharpfs z. B. die explizit große Bedeutung des „nationalstaatlichen Veto gegen alle Entscheidungen, durch die eigene Interessen verletzt werden könnten“ (326) und die häufige Verwendung von „Einstimmigkeit“ ohne Ergänzung. Eine Passage am Ende von Scharpfs Text grenzt die E(W)G-vertraglich verankerte Möglichkeit zu Mehrheitsvoten von der Politikverflechtungsfallen-Situation relativ klar ab: „Der prekäre Charakter des Problemlösungs-Modus und die allgegenwärtige Gefahr eines Rückfalls in den Bargaining-Modus, der für die Politikverflechtung charakteristisch ist, kann sich also wohl doch nur in Entscheidungs-Situationen zeigen, in denen die De-facto-Einstimmigkeit nicht abgesichert ist durch formelle Möglichkeiten der oktroyierten (hierarchischen oder Mehrheits-)Entscheidung oder durch die faktische Übermacht eines hegemonialen Partners" (Scharpf 1985: 351). Im EG-Fall der qualifizierten Mehrheit ist eine formelle Möglichkeit zur Mehrheitsentscheidung gegeben.

Gegen eine gleiche Behandlung von Einstimmigkeit und qualifizierter Mehrheit im Falle der EG würden außerdem mehrere Argumente sprechen. Erstens wären dann so gut wie alle relevanten EG-Politiken unterschiedslos Opfer der Politikverflechtungsfalle (einfache Mehrheit kommt im Vertrag auf der policy-making-Ebene fast nicht vor, daher werden praktisch alle Entscheidungen mit qualifizierter Mehrheit oder Einstimmigkeit getroffen). Zweitens würden bei der Messung an einem so wenig differenzierten Maßstab signifikante Veränderungen im Zeitverlauf übersehen. Die Einführung des Kooperations- und später des Kodezisionsverfahrens, die beide auf qualifizierter Mehrheit im Rat aufbauen, wäre bedeutungslos. Drittens belegen die empirischen Erfahrungen der EG-Sozialpolitik eindeutig, dass der Wechsel zu qualifizierter Mehrheit einen signifikanten Unterschied in der Entscheidungsdynamik ausmachte. In den 1990er Jahren wurde die überwiegende Mehrheit der sozialpolitischen Entscheidungen übrigens nicht in Anwendung von Entscheidungsverfahren mit Einstimmigkeitsregel getroffen (zum allgemeinen Trend in Richtung Mehrheitsvoten vgl. etwa H. Wallace 1996: 153). Aus all diesen Gründen setze ich in diesem Text den Übergang zu qualifizierten Mehrheitsabstimmungen im Rat mit dem Ende einer Politikverflechtungsfallensituation gleich. 
Entsprechend Scharpfs Erwartungen führten die Einstimmigkeitserfordernisse zu zahlreichen Blockaden (vgl. schon oben), und der Output an sozialpolitischen EG-Rechtsakten wurde über viele Jahre hinweg von politischen wie auch von akademischen BeobachterInnen fast durchwegs als nicht zufrieden stellend beurteilt. Dies gilt primär für die Zahl der Verabschiedungen, aber auch für die konkret vereinbarten Standards. ${ }^{13}$ Angesichts der sozialpolitischen Untätigkeit der EG in zahlreichen Bereichen, wo der Binnenmarkt mindestens indirekte Effekte auf die Sozialsysteme haben würde, bestanden vielfach Bedenken, dass Betriebe mancher Staaten fragwürdige „Wettbewerbsvorteile“ nützen könnten bzw. dass die Standards in den fortschrittlicher Ländern über „Sozialdumping “14 gefährdet seien. „The ongoing integration process necessarily affects national social policies. As integration deepens, these effects become pervasive" (Leibfried 1992: 97; in diese Richtung z.B. auch Lange 1993; Falkner 1992; Schulte 1991; Leibfried 1990).

Die Koexistenz von Frustration und Stabilität in einer Scharpfschen Politikverflechtungsfalle wurde also im Fall der EG-Sozialpolitik vor dem Maastrichter Vertrag kaum bestritten. Eine befriedigende Erklärung dieses Zustandes ist jedoch laut Wolfgang Streeck nur bei expliziter Berücksichtigung auch der politischen Ökonomie der transnationalen Politikverflechtung möglich, also deren Wechselverhältnisses mit dem System wirtschaftlicher Interessen und der dortigen Machtverteilung (Streeck 1995b: 106). In der korporatistischen transnationalen Politik konstatierte Streeck eine „Politik des leeren Stuhles“ und daraus resultierend eine Entscheidungslücke, weil die Arbeitgeberseite sich über Jahrzehnte hinweg nicht als organisierter Verhandlungs- und Vertragspartner zur Verfügung stellte (Streeck 1995b: 116f.). Die Arbeitgeber hatten „keinerlei kollektive Interessen, die als Anknüpfungspunkt für ein gegenseitiges Geben und Nehmen mit der Arbeitnehmerseite dienen könnten“ (Streeck 1995b: 117). Und weil in der Politikverflechtungsfalle der EG-Sozialpolitik schon eine Gegenstimme im Ministerrat genügte, um ein sozialpolitisches Projekt zu blockieren, musste sich die Arbeitgeberseite auch kaum Sorgen vor Regulierung durch den Ministerrat machen: ihre präferierte Option der Nichtintervention war ohnehin die Default-Lösung (siehe auch Streeck 1995a: 37, Streeck/Schmitter 1994: 207). ${ }^{15}$

Damit war die jahrzehntelang (und zwar gemessen an den verschiedensten Indikatoren, siehe unten) so geringe Problemlösungskapazität der EG-Sozialpolitik politikwissenschaftlich treffend analysiert. Tatsächlich ist festzustellen, dass trotz vielfältiger Bemühungen der EG-Kommission (vor allem ab dem Amtsantritt von Jacques Delors 1985) die regelmäßig unter dem Titel „sozialer Dialog“ abgehaltenen Treffen der zentralen drei Dachverbände

13 Für kritische Beiträge siehe etwa Adamy (1989 und 1990), Arbeitskreis Außenpolitik (1988), Aubry (1989), Beckmann/John/Wathling (1991), Betten/Harris/Jaspers (1989), Birk (1990), Steinkühler (1989), Böhme/Peressin (1990), Böttcher (1990), Breit (1988), Brok (1988), Deubner (1990), Maydell (1990), Bukold/Musiolik (1990), Chassard (1990), Däubler (1989a), Deutscher Gewerkschaftsbund (1987), Europäischer Gewerkschaftsbund (1988 und 1989), Europäisches Gewerkschaftsinstitut (1988), Franzmeyer (1988), Hoskyns (1986), Hörburger (1990), Jonckheer/Pochet (1990), Lodge (1989), Marin (1988), Observatoire Social Européen (1989), Partikel (1990), Schulte (1985), Schulz (1991), Vogel-Polsky (1989 und 1990).

14 Zu diesem Begriff vgl. Falkner (1993b).

15 Aufgrund der ,institutionalisierte(n) Entscheidungslücke im System transnationaler Interessenpolitik“ (Streeck 1995a: 122) sei auch Scharpfs Vorschlag (1994: 237), soweit als möglich auf korporatistische Prozesse zurückzugreifen um autonomieschonende aber gemeinschaftsverträgliche Regelungen zu erreichen, nicht realisierbar. 
auf Arbeitgeber- und Arbeitnehmerseite (UNICE, ${ }^{16} \mathrm{CEEP}^{17}$ und EGB ${ }^{18}$ ) zumindest mittelfristig zu keinen nennenswerten Ergebnissen führten. Bis in die 1990er Jahre waren die Hoffnungen der Kommission, die „Sozialpartner“ auf europäischer Ebene könnten ihren sozialpolitischen Vorschlägen vermehrte Legitimität gegenüber dem fast regelmäßig blockierten Ministerrat verleihen, daher vergeblich. Aber dann kam der Maastrichter Vertrag.

\section{Die Maastrichter Reform der EG-Sozialpolitik}

Obwohl die Ausgangsbedingungen dafür denkbar schlecht waren (Scharpf 1985: 348), gelang es der EG, im Sozialbereich der Politikverflechtungsfalle zu entkommen. In Maastricht wurden maßgebliche institutionelle Reformen beschlossen, die zumindest für weite Bereiche die inhärente Tendenz zur Selbstblockierung zu überwinden erlaubten.

Innerhalb der Verhandlungen zum Maastrichter „Vertrag über die Europäische Union“ (EUV) erreichte Soziales 1991/92 einen zuvor unerreichten Stellenwert auf EG-Ebene. An der Weigerung Großbritanniens, die von den anderen elf Mitgliedstaaten gewünschte und auch von anderen EG-Akteuren (v.a. EP, Kommission) erwartete Erweiterung der sozialpolitischen Kompetenzen mitzutragen, wäre beinahe die gesamte Vertragsreform gescheitert. Erst um Mitternacht des letzten Verhandlungstages konnte eine Kompromisslösung gefunden werden (Schulz 1996: 90ff.; Ross 1995: 191), welche eine zweigleisige Lösung vorsah. ${ }^{19}$ Während die sozialpolitischen Bestimmungen des EWG-Vertrages unreformiert blieben, sollte für die Mitgliedstaaten außer Großbritannien künftig eine eigene Rechtsgrundlage gelten: Das Abkommen zwischen den Mitgliedstaaten der EG mit Ausnahme des Vereinigten Königreichs und Nordirland über die Sozialpolitik stellte eine sozialpolitisch beträchtlich weiter gehende Gesetzgebungsbasis dar, was vertragliche Handlungsaufträge wie auch politisch-institutionelle Handlungsmöglichkeiten betrifft.

Als sozialpolitische Ziele wurden „der Gemeinschaft und den genannten Mitgliedstaaten " in Artikel 1 des Abkommens (jetzt Artikel 136 EGV) zugeschrieben: Förderung der Beschäftigung, Verbesserung der Lebens- und Arbeitsbedingungen, angemessener sozialer Schutz, sozialer Dialog, Entwicklung des Arbeitskräftepotenzials im Hinblick auf ein dauerhaft hohes Beschäftigungsniveau und Bekämpfung von Ausgrenzungen. Zu diesem Zweck sollen „die Gemeinschaft und die genannten Mitgliedstaaten Maßnahmen durch(führen), die der Vielfalt der einzelstaatlichen Gepflogenheiten, insbesondere in den vertraglichen Beziehungen, sowie der Notwendigkeit, die Wettbewerbsfähigkeit der Wirtschaft in der Gemeinschaft zu erhalten, Rechnung tragen“" (ebenda).

Die konkrete Verwirklichung dieser Ziele wurde dadurch maßgeblich erleichtert, dass seit Inkrafttreten des Maastrichter Vertrages im November 1993 qualifizierte Mehrheitausreicht, um „schrittweise anzuwendende Mindestvorschriften“ (Artikel 137 Absatz 2) auf

16 Europäische Vereinigung der Arbeitgeber- und Industrieverbände (Union des Confédérations de l'Industrie et des Employeurs d'Europe).

17 Europäischer Zentralverband der öffentlichen Wirtschaft (Centre Européen des Enterprises à Participaton Publique).

18 Europäischer Gewerkschaftsbund.

19 Juristisch blieben allerdings viele Details ungeklärt, und die mangelhafte Kohärenz zwischen dem Protokoll und dem Vertrag selbst wurde allgemein kritisiert (z.B. Watson 1993: 492ff.; Weiss 1992: 3; Fitzpatrick 1992: 203; Vogel-Polsky 1994: 86; Assicredito 1995). 
den folgenden zusätzlichen Gebieten zu beschließen: Arbeitsbedingungen, Unterrichtung und Anhörung der Arbeitnehmer, Chancengleichheit von Männern und Frauen am Arbeitsmarkt und Gleichbehandlung am Arbeitsplatz sowie berufliche Eingliederung der aus dem Arbeitsmarkt ausgegrenzten Personen. Besonders bemerkenswert ist dabei, dass die in der Praxis der EG-Sozialpolitik zentrale Frage der Regulierung von Arbeitsbedingungen mehrheitsfähig gestaltet wurde. Das für die Politikverflechtungsfalle entscheidende Erfordernis der Einstimmigkeit gilt seit dem Sozialprotokoll nur mehr für einige spezifische Themen. $^{20}$

Im Kontext der gängigen Entscheidungsverfahren auf Unionsebene völlig neuartig waren jene Muster, die für die Beteiligung von Arbeitnehmer- und Arbeitgebervertretungen ${ }^{21}$ vorgesehen wurden. Im Zuge einer nunmehr formalisierten Anhörung der Sozialpartner vor der Unterbreitung von Vorschlägen im Sozialbereich durch die Kommission können diese mitteilen, dass sie in Kollektivverhandlungen eintreten wollen. In diesem Fall unterbricht die Kommission den normalen Rechtsetzungsprozess. ${ }^{22}$ Kommt es zu einer Vereinbarung zwischen Arbeitgeber- und Arbeitnehmerseite, so kann diese (auf Antrag der Unterzeichner) durch einen Beschluss des Rates auf Antrag der Kommission als verbindliches EG-Recht implementiert werden. Folgt man der klassischen Definition von Philippe Schmitter (1981: 295), ${ }^{23}$ so wurde mit diesen Bestimmungen ein korporatistisches Entscheidungsmuster verankert, nach dem die maßgeblichen Interessengruppen mitverantwortliche Partner in der europäischen Politikgestaltung sind. ${ }^{24}$

Vor dem oben ausgeführten Hintergrund waren die Erwartungen diverser akademischer und wissenschaftlicher Beobachter in Bezug auf die praktische Verwirklichung der Maastrichter Neuerungen in der konkreten EG-Sozialpolitik nahe liegender Weise eher skeptisch (vgl. etwa Keller 1993 und 1997; Lange 1993; Falkner 1993a; Streeck z.B. 1994 und 1995a; Pierson/Leibfried 1995a; Traxler/Schmitter 1995a,b). Nichtsdestotrotz kam es mittlerweile tatsächlich zu mehreren Kollektivabkommen, die in der Folge vom Ministerrat in Form von EG-Richtlinien verbindlich gemacht wurden. Auf intersektoraler Ebene wurden solcherma-

20 Für Fragen der sozialen Sicherheit und des sozialen Schutzes der ArbeitnehmerInnen, für den Schutz der ArbeitnehmerInnen bei Beendigung des Arbeitsvertrages, die Vertretung und kollektive Wahrnehmung der Arbeitnehmer- und Arbeitgeberinteressen einschließlich der Mitbestimmung, die Beschäftigungsbedingungen der Staatsangehörigen dritter Länder und für finanzielle Beiträge zur Förderung der Beschäftigung und zur Schaffung von Arbeitsplätzen (Artikel 2 Absatz 3 Sozialabkommen, jetzt Artikel 137 Absatz 3 EGV). Überhaupt von der Geltung des Artikel 2 des Abkommens der Elf ausgeschlossen wurden übrigens Maßnahmen hinsichtlich des Arbeitsentgelts, des Koalitionsrechts, des Streikrechts sowie des Aussperrungsrechts (vgl. jetzt Artikel 137 Absatz 6 EGV). Sie können daher nur auf Grundlage anderer Vertragsbestimmungen einer EG-Regelung zugeführt werden (Bercusson 1995: 178).

21 In der deutschen Fassung des Vertrages ist hier von „den Sozialpartnern“ die Rede, in der englischen Fassung von „management and labour“ (vgl. Art. 3 und 4 Sozialabkommen, jetzt Artikel 137 und 138 EGV).

22 Für neun Monate; die Kommission und die Sozialpartner können jedoch gemeinsam eine Verlängerung beschließen (Artikel 3 Absatz 4 Sozialabkommen, jetzt Artikel 137 und 138 EGV).

$23, \ldots$ a mode of policy formation in which formally designated interest associations are incorporated within the process of authoritative decision-making and implementation. As such they are officially recognised by the state not merely as interest intermediaries but as co-responsible ,partners' in governance and social guidance" (Schmitter 1981: 295).

24 Um dieses Phänomen vom sektorübergreifenden „Korporatismus“ der 1970er Jahre abzugrenzen und auf die großen politikfeldspezifischen Unterschiede in der europäischen Entscheidungsfindung hinzuweisen, sollte heute besser von einer „korporatistischen Politikfeldgemeinschaft“ (corporatist policy community) gesprochen werden (Falkner 1998). 
ßen Vorschriften zum Elternurlaub, zur Teilzeitarbeit und zu befristeten Arbeitsverhältnissen beschlossen, auf sektoraler Ebene wurden auf diesem Weg Regeln über die Arbeitszeit für Seeleute verabschiedet. Darüber hinaus wurden von den einschlägigen EU-weiten Sozialpartnerverbänden weitere Abkommen, etwa über die Arbeitszeit im Eisenbahnsektor sowie in der zivilen Luftfahrt, geschlossen. All dies sind regulative Projekte, die zuvor über viele Jahre lang im Rat blockiert gewesen waren.

Auch die seit Maastricht bestehende Möglichkeit zur Verabschiedung sozialpolitischer Richtlinien in Mehrheitsabstimmungen fand in der politischen Praxis Niederschlag. ${ }^{25}$ Sogar schon vor dem Inkrafttreten des Maastrichter Vertrages (im November 1993) führe die Vereinbarung unten den damals elf Mitgliedstaaten außer Großbritannien dazu, dass seither praktisch alle Maßnahmen der EG-Sozialpolitik nicht einstimmig ${ }^{26}$ beschlossen wurden. ${ }^{27}$ Das Ende der Politikverflechtungsfalle in diesem Bereich (durch die Einführung von Mehrheitsvoten für weite Bereiche der EG-Sozialpolitik, besonders für die in der Praxis der EG-Sozialpolitik so bedeutsamen Arbeitsbedingungen) wurde in der Folge durch den Amsterdamer Vertrag (in Kraft seit Mai 1999), der die Regeln des Sozialabkommens auf alle Mitgliedstaaten ausdehnte, nur mehr bestätigt.

Dies bedeutet noch keineswegs, dass auch der output der EG-Sozialpolitik optimal wäre (vgl. dazu unten), oder dass keine harten Konflikte mehr zwischen den Delegationen ausgetragen würden. In der zweiten Hälfte der 1990er Jahre stellten aber weder das Einstimmigkeitserfordernis noch die korporatistische Entscheidungslücke als quasi institutionelle Hürden auf Unionsebene das vorrangige Problem der EG-Sozialpolitik dar. Vielmehr schien die Abwesenheit einer sowohl quantitativ als auch qualitativ weiter gehenden EG-Sozialpolitik die präferierte Option sehr vieler EG-Regierungen zu sein. Während immer seltener von der Kommission vorgeschlagene Projekte von einzelnen Delegationen entgegen den Wünschen einer Mehrheit blockiert wurden, war seit dem Maastrichter Vertrag (zumindest auf Ebene der EG-Institutionen und bis zur Jahreswende 1999/2000) ein Mangel an sozialpolitischen Visionen für die EU zu konstatieren. In dieser Zeit stand der ernsthaften Diskussion von in-

25 Eine Analyse der Mechanismen, welche es der EG-Sozialpolitik im Detail ermöglichten, trotz der wenig vielversprechenden Ausgangsbedingungen die „Fallen“ der Einstimmigkeit und der korporatistischen Entscheidungslücke zu überwinden, hätte den Rahmen dieses Beitrags gesprengt (siehe aber Falkner 2000).

26 Es ist aber darauf hinzuweisen, dass die überwiegende Mehrzahl aller Ratsbeschlüsse ohne formelle Abstimmung zustande kommt. Immerhin gibt es jedoch für die zentralen sozialpolitischen Richtlinien der letzten Jahre (Arbeitszeit, Arbeitskräfteentsendung, jugendliche Arbeitskräfte, Europäische Betriebsräte, schwangere Arbeitskräfte sowie für die schon unter dem Sozialprotokoll beschlossenen Maßnahmen in Bezug auf Elternurlaub und Teilzeitarbeit) Belege dafür, dass eine bzw. mehrere Delegationen sich entweder konkret dagegen aussprachen oder still ,übergehen“ ließen, dass sie aber bei Bestehen eines Einstimmigkeitserfordernisses mit sehr großer Wahrscheinlichkeit blockiert hätten (vgl. Falkner 1998: 69).

27 Kaum war die Tür für Mehrheitsentscheidungen prinzipiell geöffnet, vollzog die Praxis der EG-Sozialpolitik das Ende des Einstimmigkeitserfordernisses außerdem schon quasi im voraus. Das sogenannte „Vertragsgrundlagenspiel“ (Treaty-base game, M. Rhodes 1995: 99) wurde ab diesem Zeitpunkt intensiv angewandt, und man zwang Großbritannien über eine extensive Interpretation der Bestimmung über Sicherheit und Gesundheitsschutz der Arbeitskräfte (Artikel 118a EWGV, jetzt Artikel 138 EGV), die einzig innerhalb des Sozialkapitels Beschlussfassung mit qualifizierter Ratsmehrheit erlaubte, auch für den weiteren Bereich der Arbeitsbedingungen zu akzeptieren. Einer Klage des Vereinigten Königreichs gegen die so beschlossene Arbeitszeitrichtlinie (93/104/EWG, ABl. 93/L 307/18) wurde vom EuGH am 12 November 1996 im wesentlichen nicht stattgegeben (Rechtssache C-84/94). 
novativen sozialpolitischen Projekten auf Unionsebene vor allem ein mainstream von neo-liberalen und monetaristischen Wirtschaftsschulen einerseits und von national orientiertem ,Subsidiaritätsdenken' andererseits entgegen. Damit waren die primären Hindernisse für einen Ausbau der sozialen EG-Dimension eher ideelle denn institutionelle Aspekte. ${ }^{28}$

Bevor am Ende dieses Beitrags auf jüngste, innovative Entwicklungen eingegangen wird (deren vollständige Ausformulierung auf Unionsebene sowie der praktische Umsetzung allerdings noch ausstehen), sollen verschiedene Maßstäbe für den bestehenden acquis communautaire der EG-Sozialpolitik diskutiert werden.

\section{Die soziale Dimension der europäischen Integration: Bewertungsmaßstäbe und Erfolg}

Die Entscheidungsfähigkeit der EG in Fragen der Sozialpolitik wurde, wie oben ausgeführt, in den 1990er Jahren signifikant verbessert. Im Sinne der letztlich praktisch relevanten Frage nach der Problemlösungskapazität der EG im Sozialbereich ist allerdings nicht nur relevant, ob entschieden wird, sondern auch, welche Ziele sich die EG-Sozialpolitik überhaupt steckt, was also problematisiert wird. ${ }^{29}$

Die Ansprüche akademischer wie politischer Beobachter bzw. Akteure in Hinblick auf die einschlägige Rolle der EG waren und sind höchst unterschiedlich, wobei zumindest drei Faktoren eine ausschlaggebende Rolle spielen: das grundlegende Verständnis einer adäquaten Aufgabenteilung zwischen der nationalen und der supranationalen Ebene, die Position in Hinblick auf Marktfreiheit versus politischer Steuerung, sowie ein engeres oder weiteres Verständnis von Sozialpolitik (im Unterschied etwa zu Beschäftigungs- oder allgemeiner Wirtschaftspolitik). Im Folgenden werden vier mögliche Beurteilungsmaßstäbe für den sozialpolitischen acquis der EG diskutiert. Sie sind aus der einschlägigen juristischen und sozialwissenschaftlichen Debatte bekannt, wurden aber in der Regel dort nur implizit herangezogen und nicht ausführlich argumentiert. Da die Kriterienbildung den ,Leitmotiven' der breiten akademischen und öffentlichen Diskussion der 1980er und 90er Jahre folgt, kommen sowohl formale (Kommissionsvorlagen) als auch materielle Kriterien (z.B. Schließung der durch das Binnenmarktprogramm bedingten arbeitsrechtlichen Lücken) vor. ${ }^{30}$ Die jeweils formulierten Anforderungen sind unterschiedlich, wobei im Zeitverlauf die ver-

28 Dies galt selbst für jene Einzelthemen, für die das Einstimmigkeitserfordernis weiter besteht. So waren etwa im diesbezüglich wohl wichtigsten Bereich der sozialen Sicherheit kaum Blockaden zu verzeichnen, weil es schon an relevanten Projekten mangelte. Wenn Hindernisse weniger EG-institutionelle als ideelle sind, so bedeutet dies natürlich nicht, daß sie deshalb schon leichter überwunden werden können.

29 Davon wiederum zu trennen ist die Dimension der empirischen Zielerreichung, die in diesem Beitrag nicht behandelt werden kann. Dazu ist nicht nur notwendig, daß erstens ein sozialpolitisches Programm tatsächlich in Beschlüsse umgesetzt wird und daß die beschlossenen Maßnahmen zweitens die wahrgenommenen Probleme tatsächlich zu lösen geeignet sind, sondern darüberhinaus auch noch, daß drittens die praktische Umsetzung und Anwendung der Maßnahmen in den Mitgliedstaaten zufriedenstellend durchgeführt wird. Es geht dabei also um die ,Problemlösungsfähigkeit der Sozialpolitik im Mehrebenensystem', nicht mehr nur um jene der EG-Sozialpolitik im engeren Sinne.

30 Im Unterschied zu bekannten älteren Beiträgen zur EG-Sozialpolitik wurde die Unterscheidung sozialpolitischer EG-Aktivitäten nach der Form der EG-Intervention - also regulativ/distributiv/redistributiv (vgl. Majone 1993) bzw. auch positive/negative Integration (Scharpf z.B. 1997c und 1998b) - in diesem Fall nicht als zentrales Kriterium gewählt. 
gleichsweise weiterreichenden an Bedeutung zunahmen. Dies steht nicht zuletzt auch in Zusammenhang damit, dass die ursprünglich engen sozialpolitischen Zielsetzungen der EG mittlerweile ausgedehnt wurden und nunmehr etwa auch die Förderung der Beschäftigung, angemessenen sozialen Schutz, die Entwicklung des Arbeitskräftepotenzials im Hinblick auf ein dauerhaft hohes Beschäftigungsniveau und die Bekämpfung von Ausgrenzungen umfassen (Artikel 136 EGV). Damit wurde - zumindest von der Vertragsgrundlage her sozialpolitische Gestaltung im weitesten Sinne ermöglicht. Im Vergleich dazu nimmt sich die bislang fast ausschließlich praktizierte arbeitsrechts-zentrierte Ausrichtung der EG-Sozialpolitik, die auch den ersten beiden Bewertungsmaßstäben zu Grunde liegt, umso enger aus.

\subsection{Die Behebung arbeitsrechtlicher Lücken}

Im EG-Binnenmarkt stand die weiterhin nur nationale Geltung der einzelnen Arbeitsrechtsordnungen plötzlich einem transnationalen Wirtschaftsraum gegenüber. Bedenken, dass es zu einem durch die Liberalisierung direkt induzierten Abbau von Arbeitnehmerrechten kommen könne, wurden vor allem von deutschen Arbeitsrechtsexperten und Gewerkschaftern geäußert (siehe vor allem Däubler 1989b: 150, 1989c: 66f., 1996: 154; und die Beiträge in Breit 1988).

Das wichtigste Beispiel für einen solchen Fall war die Frage, welches Arbeitsrecht für in andere EG-Länder entsandte Arbeitskräfte gelten solle. Entsendungen wurden ja durch die praktische Verwirklichung der Dienstleistungsfreiheit immer häufiger. Vor dem Hintergrund, dass etwa im Bausektor 50\% der Kosten durch die Löhne entstehen, ergab sich für Länder mit hohen Arbeitskosten, besonders für die Bundesrepublik, ein potenziell stark verzerrender Effekt zu Gunsten von Anboten durch aus dem billigeren Ausland entsendende Unternehmen (z.B. Däubler 1996: 155). Lange Zeit schien es, als könnten sich die Regierungen im Ministerrat nicht auf eine einschlägige EG-rechtliche Maßnahme einigen. Aber im Dezember 1996 kam es, fünfeinhalb Jahre nach dem entsprechenden Kommissionsvorschlag, schließlich doch zur Verabschiedung (RL 96/71/EG, ABl 97/L 18/1).

Wie Wolfgang Streeck ausführt, ist an der Entsenderichtlinie wegweisend, dass das Interesse der Mitgliedstaaten an einer Stabilisierung ihrer jeweiligen nationalen Sozialordnungen anerkannt und „der Schutz der nationalen Sozialpolitik vor dem Diktat des europäischen Wettbewerbsrechts" (1996: 14) sichergestellt wurde. Allerdings müssen die Mitgliedstaaten dabei nicht bestimmte materielle Standards einführen. Wie schon seit Jahrzehnten üblich in Hinblick auf die Gleichbehandlung der Geschlechter und die Gleichstellung von Wanderarbeitskräften in den einzelnen Sozialversichungssystemen, wird „nur“ die Nicht-Diskriminierung einer Personengruppe, hier konkret der aus anderen Mitgliedstaaten entsandten Arbeitskräfte, festgelegt. Trotzdem ist dies vor dem Hintergrund der langjährig berechtigten Blockadebefürchtungen (siehe noch Däubler 1996: 154f.) als ein Beleg für Handlungskapazität der EG anzusehen. Die Richtlinie wurde auf Grundlage des Kodezisions-Verfahrens mit dem Europäischen Parlament (Artikel 189b EGV, jetzt Artikel 251 EGV) mehrheitlich verabschiedet und verpflichtet die Mitgliedstaaten sicherzustellen, dass zentrale Arbeitsbedingungen und der Lohn entsandter Arbeitskräfte nach dem Arbeitsrecht des Arbeitsortes gewährt werden. ${ }^{31}$

31 Besonders Großbritannien, Portugal, Griechenland und Dänemark hatten sich lange Zeit gegen das 
Ein ähnlicher Fall ist die Richtlinie über europäische Betriebsräte in grenzüberschreitend tätigen Konzernen (RL 94/45/EWG, ABl. 94/L 254/64). Weil im Zuge der wegfallenden Marktgrenzen (v.a. auch für Kapital) stetig mehr Firmen zu „Multinationalen“ wurden, blieb die in nationalen Gesetzen geregelte Arbeitnehmermitbestimmung immer öfter praktisch wirkungslos: die maßgeblichen Entscheidungen wurden im Ausland in der Konzernzentrale getroffen, für welche die nationalen Regeln nicht galten. Deshalb schien eine EG-weite Betriebsräteregelung notwendig, um zur Arbeitnehmerinformation und -konsultation im Binnenmarkt sicherzustellen. ${ }^{32}$ Trotz berechtigter Kritikpunkte an spezifischen Details der Richtlinie (vgl. v.a. Streeck 1997) war ihr Zustandekommen ein für viele überraschendes Zeichen für die Entscheidungskraft der post-Maastricht-EG auch in sozialpolitischen Belangen. Die Verabschiedung erfolgte im Kooperationsverfahren (das qualifizierte Mehrheit erlaubt) unter den Bestimmungen des sozialpolitischen Abkommens der Mitgliedstaaten außer Großbritannien. ${ }^{33}$

Folgen wir also dem (vor allem in juristischen Kreisen sozusagen als ,Minimalanforderung' an eine, soziale Dimension' der EG diskutierten) Kriterium, dass die EG-Sozialpolitik zumindest jene unmittelbaren Arbeitsrechtsprobleme lösen muss, die sich aus dem Binnenmarktprogramm ergaben, so ist festzustellen, dass sich entgegen häufigen Befürchtungen die Handlungskapazität der EG eher größer denn erwartet darstellt. ${ }^{34}$

\subsection{Kommissionsvorschläge versus verabschiedete Rechtsakte}

Ein etwas größere Anforderungen stellender Beurteilungsmaßstab für Erfolg oder Misserfolg der sozialen Dimension der EG ist der Vergleich zwischen den vorgeschlagenen und den tatsächlich vom Rat ${ }^{35}$ verabschiedeten Bestimmungen. Diese Variante setzt im Unterschied zu den anderen an einem formalen Kriterium an. Ein großes Differenzial zwischen Kommissionsvorschlägen und Ratsbeschlüssen würde auf einen Reformstau hindeuten, also auf eine empirische Entscheidungslücke in der Praxis der EG-Sozialpolitik. Ein Charakteristikum dieses Maßstabes ist, dass der Europäischen Kommission ein entscheidender Stellenwert im Sinne von Definitionsmacht eingeräumt wird. Ihrem Ermessen obliegt es ja, ob ein sozialpolitisches Vorhaben als inhaltlich sinnvoll und politisch opportun eingestuft wird. Nur in diesem Falle wird sie es auf die offizielle Agenda der EG-Sozialpolitik setzen und eine Blockade vor dem kritischen Blick der Öffentlichkeit riskieren. Was aber von der Kommission nicht auf die politische Tagesordnung gesetzt wird, bleibt in Hinblick auf die Problemlösungsfähigkeit der EG-Sozialpolitik hier außer Betracht.

Vorhaben ausgesprochen (Agence Europe, 29.3.1996: 9), zuletzt dürften nach Informationen aus „gewöhnlich wohlinformierten Kreisen" Großbritannien und Portugal dagegen gestimmt haben.

32 Obwohl dabei die Arbeitnehmer-Mitbestimmung im engeren Sinne nicht berührt wird, waren die europäischen Betriebsräte von Gewerkschaftsseite als besonders vordringliches Anliegen bezeichnet worden, weil sie einen organisatorischen Unterbau für die grenzüberschreitende Kooperation der Arbeitnehmervertretungen auf Unternehmensebene darstellen.

33 Zur Vorgeschichte siehe z.B. Falkner (1996).

34 Als gesellschaftsrechtliche Maßnahme mit Bezug zu Arbeitnehmerrechten, wo noch keine Einigung auf EG-Ebene gelungen ist, bleibt die Arbeitnehmermitbestimmung in der „Europäischen Aktiengesellschaft" zu nennen. Diese wurde, gerade wegen der offenen Mitbestimmungsfrage, noch nicht eingerichtet. Das Problem ist, dass es hier um grundlegende Systemunterschiede in der Arbeitnehmerbeteiligung geht und die Grundpositionen selbst innerhalb der Gewerkschaften unvereinbar scheinen, weil der Export des weitestgehenden deutschen Modells umstritten ist.

35 Teils in Zusammenarbeit mit dem EP. 
Auch an diesem Maßstab gemessen zeigt sich die EG handlungsfähiger als vielfach noch bis vor kurzem erwartet wurde. Mittlerweile sind alle damals neuen Vorschläge des sozialpolitischen Aktionsprogramms zur Umsetzung der Sozialcharta aus 1989 verabschiedet (für einen Überblick siehe Falkner 1998: 69), und auch die zuvor langfristig blockierten älteren Vorschläge aus dem Aktionsprogramm 1974 sind - soweit sie je konkret vorgelegt wurden bis auf sehr wenige Detailaspekte ${ }^{36}$ beschlossene Sache. Selbst in der so kontroversiellen Frage der Beweislastumkehr in Fällen geschlechtsspezifischer Diskriminierung wurde ein gemeinsamer Nenner gefunden.

$\mathrm{Zu}$ betonen ist, dass die beschlossenen Maßnahmen über die Schließung der oben besprochenen Rechtslücken weit hinausgehen. Vielmehr wurde auch eine Anzahl sozialpolitischer Mindeststandards zu (meist arbeitsrechtlichen) Detailfragen beschlossen. Dabei spielte eine direkte Betroffenheit durch die Wirtschaftsintegration keine Rolle: es wurden auch Standards Gegenstand von EG-Regulierung, die nur national aktive Firmen ebenso betreffen wie grenzüberschreitend tätige. So hat etwa das Ausmaß des Elternurlaubs per se nichts mit dem europäischen Binnenmarkt zu tun - außer, dass es zu den gesamten Arbeitskosten in jedem Land beitragen kann, und diese wiederum in einem Binnenmarkt noch stärker wettbewerbsrelevant werden. ${ }^{37}$ Die Arbeitskosten setzen sich jedoch aus vielfältigen Einzelposten zusammen (direkte Lohnkosten pro Stunde, Arbeitszeit, Sozialversicherungsbeiträge etc.), von denen von der EG wiederum bei weitem nicht alle angeglichen wurden. ${ }^{38}$ Diese EG-weiten sozialen Mindeststandards im Arbeitsrecht sind also eher als ein eigenständiges Interventionsziel der EG denn als direkt marktbedingte Maßnahmen zu sehen. Insofern hat sich in den vergangenen Jahren die EG-Sozialpolitik doch beträchtlich weiter entwickelt, als noch Ende der 1980er Jahre zu erwarten stand. Damals wurde zwar die so genannte „EG-Sozialcharta über soziale Grundrechte der Arbeitnehmer" von elf Staats- und Regierungschefs beschlossen. Weil Großbritannien aber abseits blieb, stellte dieser feierliche, aber unverbindliche Akt streng genommen nicht einmal EG-Recht dar, und die Verwirklichung der im Aktionsprogramm der Kommission vorgesehenen EG-Richtlinien war vielfach bezweifelt worden.

Der Maßstab der abgeschlossenen Rechtsetzungsverfahren hat allerdings zwei „blinde Flecken“. Erstens wird nichts über die Qualität des beschlossenen EG-Sozialrechts ausgesagt. Die Problemlösungskapazität wird ja nach einem formalen Kriterium beurteilt. Ob also tatsächlich Probleme gelöst wurden, bleibt als inhaltliche Frage offen. Allerdings ist in vielen Bereichen die tatsächliche Wirkung auf die soziale Lage in den Mitgliedstaaten nicht nur von den EG-Richtlinien abhängig (für ungelöste Probleme ist diese daher nicht unbedingt bzw. nicht allein verantwortlich zu machen). Im vergangenen Jahrzehnt wurden auch in der EG-Sozialpolitik innovative regulative Muster eingeführt, die „autonomieschonend und gemeinschaftsverträglich" (Scharpf 1994) scheinen. Wesentlich ist dabei, dass einerseits nur Mindestvorschriften gemacht werden, während die Mitgliedstaaten weiterhin strengere Normen aufrechterhalten und einführen können. Darüber hinaus werden EG-

36 Vor allem ist hier noch eine Gemeinschaftsmaßnahme gegen die sexuelle Belästigung am Arbeitsplatz zu nennen.

37 Weil die Kapitalverkehrsfreiheit, die Niederlassungsfreiheit und auch die Dienstleistungsfreiheit den Unternehmen die Auslagerung ihrer Tätigkeiten in andere Länder erleichtern.

38 Die Löhne selbst sind bis heute von einer Beschlussfassung im Rahmen der Sozialvorschriften des EGV überhaupt ausgenommen. 
weit nur Rahmenbestimmungen beschlossen, die von den einzelnen Ländern spezifiziert werden. Die dabei praktizierte Subsidiarität ist eine doppelte, nämlich regionale und funktionale: die Einbeziehung der Sozialpartner wird auf allen Ebenen nicht nur zugelassen, sondern sogar angeregt (vgl. z.B. Falkner 1996).

Während Detailstudien zur praktischen Umsetzung noch ausstehen, wurde dieser neue Gesetzgebungsstil in der Literatur auf abstrakter Ebene unterschiedlich beurteilt. Positiv kann hervorgehoben werden, dass solchermaßen gesellschaftliche Akteure zur Selbstregulierung und kreativen Problemlösung auch auf unterster Ebene angeregt werden, dass es sich quasi um eine Form des Regierens im Netzwerk handelt (vgl. Kohler-Koch 1996: 370). Auf der anderen Seite scheint die Warnung berechtigt, dass solche Beschlüsse weniger eine Lösung des regulativen Irrgartens der divergenten nationalen Sozialpolitiken in der EU bringen (Rhodes 1995) denn einen sozial schädlichen Neo-Voluntarismus darstellen könnten (Streeck 1995a). Letztendlich muss aber in detaillierten Vergleichsstudien quer durch das Mehrebenensystem erhoben werden, ob der neue „policy style“ im Endergebnis nicht nur autonomieschonend und gemeinschaftsverträglich, sondern auch sozialverträglich ist. ${ }^{39}$

Zweitens ist am Maßstab der Kommissionsvorschläge verglichen mit dem output des Ministerrates auch problematisch, dass er für die Zukunft weniger praktikabel scheint als in der Vergangenheit. Die Kommission verfolgt seit einigen Jahren eine äußerst zurückhaltende Politik beim Vorschlag neuer sozialpolitischer Maßnahmen. So enthielten die sozialpolitischen Aktionsprogramme für die Phasen 1995-1997 und 1998-2000 kaum neue gesetzgeberische Initiativen, was vielfach kritisiert wurde (z.B. Visser 1997: 12; Ross 1997: 14). Vielmehr lag die Betonung (neben dem Abschluss älterer Vorhaben) auf verbesserter Umsetzung und Anwendung sowie auf Aktualisierung und Erneuerung von bestehenden Vorschriften (KOM[98] 259: 5).

\subsection{Europäisierung, um EG-induzierten Sozialabbau der Mitgliedstaaten zu vermeiden}

Soziale Problemstellungen können aus dem Binnenmarktprogramm auch weit über Lücken im arbeitsrechtlichen Bereich hinaus entstehen. Sie sind allerdings viel schwieriger abzusehen und einzuschätzen als letztere (Platzer 1996: 30). So haben die durch das Binnenmarktprogramm stark beschleunigten ökonomischen Umstrukturierungsprozesse wohl größere Friktionen am Arbeitsmarkt hervorgerufen, als ohne die so induzierte Welle von mergers and acquisitions entstanden wären. Und die relativ kurzfristige Sanierung der Staatshaushalte unter dem Diktat der Maastrichter Konvergenzkriterien für die Wirtschafts- und Währungsunion hat die Mitgliedstaaten zwar nicht rechtlich gezwungen im Sozialbereich Kürzungen vorzunehmen, es ihnen aber politisch nahe gelegt.

Doch sind Kürzungen etwa im Gesundheitswesen oder Personaleinsparungen im öffentlichen Dienst „Probleme“, anhand derer die Problemlösungskapazität der EU gemessen werden sollte? Zumindest im dominanten politischen Diskurs wurden die diversen nationalen Sparpakete eher als Teile von Problemlösungen angesehen. Die Eindämmung der Haushaltsdefizite wurde in den vergangenen Jahren als das politisch-ökonomische Hauptproblem der EU-Mitgliedstaaten betrachtet. Obwohl vereinzelt in politischen Protestveranstal-

39 Ein einschlägiges Forschungsprojekt unter Leitung der Autorin läuft gerade am Max-Planck-Institut für Gesellschaftsforschung, Köln. 
tungen gegen Sparpakete die EU-Politik für diverse Kürzungen (mit)verantwortlich gemacht wurde, waren kaum Rufe nach einer Problemlösung durch die Union zu hören. Dies hat wohl nicht zuletzt damit zu tun, dass es hier letztlich auch um Wertentscheidungen einzelner Staaten/Regierungen geht, bei welchen Budgetposten mit Kürzungen angesetzt werden soll, und dass eine Intervention „von oben“ hier aus diesem Grunde nicht unbedingt adäquat scheint. ${ }^{40}$

Anders ist dies allerdings dann, wenn Dumpingaspekte ins Spiel kommen. Zwar bestehen trotz Binnenmarkt und WWU weiterhin nicht zu unterschätzende nationale Spielräume auch im Sozialbereich (für unterschiedliche Dynamiken je nach spezifischer Interventionsform vgl. Scharpf 1997a,b,c und 1998a). Aber: Im verschärften Wettbewerb im EG-Binnenmarkt (innerhalb dessen die EG-Länder ja den ganz überwiegenden Teil ihrer Exporte abwickeln) zahlen sich überdurchschnittliche Kürzungen im Sozialbereich als Entlastung der Arbeitgeber jedenfalls in Form erhöhter Wettbewerbsfähigkeit aus (Busch 1997: 9). Angesichts dessen wurden von belgischen bzw. deutschen Wissenschaftern zwei verschiedene Modelle vorgeschlagen, um durch eine sozialpolitische Koordinierung eine „Abwertungsspirale“ zwischen den heute zu „Wettbewerbsstaaten“ (Streeck 1996) mutierten Mitgliedsländern zu unterbinden.

Das ältere Modell der „Sozialschlange“ (Dispersyn und Van der Vorst 1990) sieht für jede soziale Leistung die Festlegung eines mittleren EU-Niveaus vor. Binnen eines bestimmten Zeitraums sollen die Länder, deren Ausgaben von diesem Wert nach unten abweichen, diese erhöhen, während die nach oben abweichenden Staaten stillstehen sollen (bzw. bei einer Anhebung über einen Transfermechanismus eine vergleichbare Erhöhung in den Partnerländern finanzieren müssen). Wie Klaus Busch (1998: 23) unterstreicht, lässt dies nicht nur großen politischen Widerstand erwarten, sondern eröffnet unter Umständen auch die Möglichkeit, dass Staaten mit Abweichungen nach unten ihre Standards bewusst langsam anheben, um Transfers zu erhalten. Deshalb entwickelte Busch in Zusammenarbeit mit einer Kommission beim SPD-Bundesvorstand einen verbesserten Vorschlag. Dieses „Korridormodell“ baut darauf auf, dass in den EU-Staaten eine starke Korrelation zwischen ökonomischem Entwicklungsniveau und Sozialleistungsquote besteht (vgl. auch Scharpf 1998b: 47f.). Es wird daher empfohlen, in einem „sozialen Stabilitätspakt“ offiziell auf Unionsebene zu beschließen, an diesem engen Zusammenhang auch in der WWU festzuhalten. In drei bis vier einander stark ähnlichen Staatengruppen sollen dann jeweils Bandbeiten (Korridore) für die Sozialleistungsquoten festgelegt werden, um Dumping zu verhindern (bei der Verteilung der Ausgaben auf einzelne Sozialleistungen hätten nach diesem Modell weiterhin die einzelnen Mitgliedstaaten freie Wahl). Zentral ist, dass zwar weiterhin unterschiedliche Finanzierungsstrukturen der Sozialsysteme bestehen bleiben könnten, dass aber ein fixer Anteil der Arbeitgeberbeiträge zu den Finanzierungskosten der Sozialsysteme festgelegt werden sollte (Busch 1998: 22 nennt 50\%) um sicherzustellen, dass die einschlägige Kostenbelastung der Unternehmen im Binnenmarkt vergleichbar ist.

40 Es scheint in diesem Lichte politisch-taktisch verständlich, dass auf nationaler Ebene unterliegende Kräfte nach einem Eingriff der EU rufen mögen. Es ist jedoch weder funktional (die Union kann nicht „besser" entscheiden, wie viel Mittel in die Invaliditäts- oder aber in die Arbeitslosenversicherung gehen sollen) noch politisch-legitimatorisch (bekanntlich kann die EU noch schwerlich als Wertegemeinschaft bezeichnet werden) leicht zu argumentieren, warum hier einzelstaatliche Kompetenzen europäisiert werden sollten. 
Es kann hier nicht auf die Details der vorgeschlagenen Modelle eingegangen werden, die zumindest auf den ersten Blick autonomieschonend, gemeinschafts- und sozialverträglich scheinen. Zentral in unserem Zusammenhang ist jedoch die Feststellung, dass die Problemlösungskapazität der Union beträchtlich nachhinkt, wenn ein Ausgleich all jener Dumpingtendenzen gefordert wird, welche vom Binnenmarkt und der WWU in Gang gesetzt wurden bzw. werden könnten. Modelle wie die oben kurz beschriebenen sind bislang noch Gegenstand fast ausschließlich akademischer Auseinandersetzungen geblieben.

\subsection{Gestaltend-reformerische Sozialpolitik (im weitesten Sinne) auf europäischer Ebene}

Eine Unterbindung von Sozialdumping in einzelnen Ländern entspricht jedoch in gewissem Sinne erst der Erhaltung des Status quo ante vor der Marktöffnung. Darüber hinaus wäre aber auch aktiv sozialreformerische Intervention denkbar, welche genuin supranationale Handlungsspielräume und Problemlösungspotenziale, etwa in den Bereichen Umverteilung und Arbeitsplatzschaffung, offensiv nützen könnte.

Angesichts des undifferenzierten Schlagwortes der „Globalisierung“ wird häufig übersehen, dass rund die Hälfte des Welthandels innerhalb der großen Blöcke stattfindet (z.B. Tichy 1998a: 50 mit Daten und weiteren Nachweisen). Dies gilt in besonderem Ausmaß für die EU, wo der Anteil des Binnenhandels am gesamten Außenhandel zwischen Anfang der 1960er und Mitte der 1980er Jahre von nur 38\% auf 80\% anstieg (Tichy 1998a: 51). Auch bei den gesamten Waren-, Dienstleistungs- und Direktinvestitionsströmen wickeln die EU-Länder mittlerweile den ganz überwiegenden Großteil untereinander ab. Die EU als Wirtschaftseinheit ist also keine kleine offene Wirtschaft (wie es die einzelnen Mitgliedstaaten sind), sondern „eine große, weitgehend geschlossene: Ihre Außenwirtschaftsquote beträgt nicht 50\% wie die der Einzelstaaten, sondern bloß 10\%“" (Tichy 1998b: 231; vgl. auch Busch 1998: 25).

Wenngleich die negative Wirkung besonders der Kapitalmarktliberalisierung auf die Handlungsfähigkeit staatlicher Gebilde in den 1990er Jahren hier nicht unterschätzt werden soll, ist dies doch als ein deutlicher Indikator dafür zu betrachten, dass auf europäischer Ebene im Prinzip auch produktionskostenerhöhende Sozialstandards reguliert und im globalen Wettbewerb verteidigt werden könnten (Busch 1998: 25). In gewissem Masse könnte so wohl auch der Tendenz gegengesteuert werden, dass die Kapitaleinkommen immer mehr entlastet und die Arbeitseinkommen stärker belastet werden (z.B. Scharpf 1997a: 26). Es gibt Überlegungen dahingehend, dass selbst zusätzliche Staatsausgaben überwiegend in der Region bleiben würden und sich über Multiplikatorwirkungen weitgehend selbst finanzieren könnten (Tichy 1998b: 231). Europäisch-gesamtwirtschaftlich betrachtet wurde auch eine restriktive Lohnpolitik, die in den einzelnen Mitgliedstaaten unter den Bedingungen der WWU ökonomisch durchaus rational scheinen mag (vgl. auch Busch 1993, 1994), als nicht zielführend bezeichnet: die restriktive Wirkung über den Kaufkraftschwund der Haushalte wird als groß eingeschätzt, während bei expansiver Lohnpolitik aber nur wenig über „Auslandsnachfrage“ abfließen würde (Tichy 1998b: 232). Wenngleich fraglos ist, dass die geeigneten institutionellen Vorgaben dafür noch nicht bestehen (Tichy 1998b: 233; Scharpf 1998a: Fn. 19), wurden also diskussionswürdige Argumente dahingehend präsentiert, dass selbst eine Art von „Euro-Keynesianismus“ ökonomisch sinnvoll durchführbar 
sein könnte, die durch offene Grenzen auf nationaler Ebene praktisch verunmöglicht wurde.

Jegliche Detailargumentation erübrigt sich aber an dieser Stelle schon deshalb, weil die Beurteilung der Leistung der EU nach diesem Kriterium - dem inhaltlich weitreichendsten der hier diskutierten - ganz offensichtlich negativ ist. Wieder ist zu betonen, dass es schon an einer intensiven Diskussion über diese und ähnliche Optionen auf Unionsebene mangelt, nicht nur an politischem output.

\section{Ausblick: Eine neue Rolle für die EG-Sozialpolitik im 21. Jahrhundert?}

Wie oben ausgeführt wurde, haben die institionellen Reformen des Maastrichter und des Amsterdamer Vertrags die (eng definierte) Beschlusskraft der EG in der „sozialen Dimension " in den 1990er Jahren beträchtlich verbessert. Im Unterschied zur von Politikverflechtungsfalle und korporatistischer Entscheidungslücke geprägten Vor-Maastricht-Situation sind heute sowohl sozialpolitische Mehrheitsentscheidungen als auch die Einbeziehung der zentralen Interessenverbände der Arbeitnehmer- und Arbeitgeberseite in die Gestaltung europäischer Sozialpolitik gängige Praxis.

Gemessen an vergleichsweise engen, arbeitsrechts-zentrierten Standards stellt sich infolge dessen die EG-Sozialpolitik mittlerweile relativ erfolgreich dar: Wesentlichen, unmittelbar durch das Binnenmarktprogramm geschaffenen arbeitsrechtlichen Problemstellungen wurde nach jahrelangen Verhandlungen letztendlich gemeinschaftlich begegnet. Auch gemessen an den offiziell dem Rat übermittelten Kommissionsvorschlägen ist der output der sozialen Dimension heute weitaus zufrieden stellender, als noch Anfang der 1990er Jahre unter den Bedingungen der Politikverflechtungsfalle zu erwarten stand. Demgegenüber ist allerdings festzustellen, dass die EG keine maßgeblichen Schritte gesetzt hat, um den laufend (und nicht zuletzt durch die EG-Liberalisierungen im Binnenmarktprogramm) erhöhten Druck auf die sozialen Standards in den Mitgliedstaaten auszugleichen und damit einem kompetitiven Abbau sozialer Standards vorzubeugen. Ein noch stärkeres Defizit ist zu konstatieren, wenn als Kriterium für die Beurteilung der sozialen Dimension der europäischen Integration die Forderung erhoben wird, dass die heute höchstens noch auf europäischer Ebene gegebenen Gestaltungspotenziale gegenüber (welt-)wirtschaftlichen Wettbewerbs„Zwängen“ sozialgestaltend genützt werden sollten, etwa um durch expansive Fiskalpolitik gezielt Wachstum und Beschäftigung zu fördern oder um der laufenden Umverteilung zu Gunsten der (v.a. Finanz-)Kapitaleigentümer gegenzusteuern.

Am Horizont zeichnet sich jedoch mittlerweile eine ganz neue Rolle für die EG-Sozialpolitik ab: jene eines Motors und zugleich eines Korsetts im Zuge der Umgestaltung der nationalen Sozial-, Beschäftigungs- und Bildungssysteme. Die Grundzüge für diese neue Funktion der EG wurden im Bereich der Beschäftigungspolitik entwickelt, wo in der Folge des Europäischen Rats von Essen (1994) sukzessive ein Koordinations- und Monitoringsystem eingerichtet und schließlich im Amsterdamer Vertrag primärrechtlich verankert wurde. Es geht dabei nicht um die, Vergemeinschaftung' dieses Politikfeldes im Sinne einer Verschiebung von legislativen Kompetenzen auf die Unionsebene, sondern um die Auslösung und Förderung von dezentralen Reformprozessen. Auf EG-Ebene werden jährliche Leitlinien beschlossen, deren Umsetzung im Detail der nationalen Ebene überlassen wird. 
Allerdings müssen von allen Mitgliedstaaten regelmässige Berichte vorgelegt und von Kommission und Rat begutachtet werden, sodass peer pressure als disziplinierender Faktor zum Einsatz kommt und zur Entblockierung nationaler Reformprozesse beitragen soll.

Angesichts dessen, dass die aktuelle Situation der einzelnen europäischen Wohlfahrtsstaaten heute bei gestiegenem Reformbedarf (aus internen wie externen Gründen) oftmals durch politische Reformunfähigkeit geprägt ist, wird dieses Modell zunehmend auch für andere Bereiche als viel versprechend betrachtet. So fasste der Europäische Rat von Lissabon im Dezember 1999 ähnliche Vorgangsweisen auch für die Bereiche Sozialversicherung, Kampf gegen die Ausgrenzung sowie Bildung ins Auge. Ziel ist ,a new open method of coordination at all levels, coupled with a stronger guiding and coordinating role for the European Council to ensure more coherent strategic direction and effective monitoring of progress" (Konklusionen der Präsidentschaft, Punkt 7). Vorerst sollen eingehende Berichte der Kommission und einer hochrangigen Arbeitsgruppe zur sozialen Sicherheit zur Lage in den einzelnen Bereichen erarbeitet werden, die auch quantitative und qualitative Indikatoren sowie benchmarks enthalten sollen. In der Folge könnte es wieder zu weit gefassten EG-Vorgaben, zu nationalen Aktionsplänen und zum Austausch von Informationen und bestpractice kommen.

Der Erfolg dieser neuen (im Vergleich zu den beiden im Abschnitt V letztgenannten Maßstäben für die EG-Sozialpolitik minimalistischen) Strategie ist zum gegenwärtigen Zeitpunkt nicht abzusehen. Selbst im Beschäftigungsbereich ist noch offen, ob es erstens zu mehr als kosmetischen Anpassungen kommt und ob diese zweitens tatsächlich zu einer Senkung der Arbeitslosigkeit führen. Jedenfalls garantiert diese neue Form voluntaristischer EG-Sozialpolitik (Streeck 1995a) schon vom Ansatz her kein gemeinschaftliches Gegengewicht zu den Kräften des Wettbewerbs, mit denen die einzelnen Länder konfrontiert sind. Sie könnte daher letztlich zwar den - auf nationaler Ebene exekutierten - Umbau von den traditionellen schutzorientierten Sozialsystemen hin zu einem angebotsseitigem Egalitarismus beschleunigen helfen (siehe dazu im Detail Streeck 1999), ohne aber die früher national gegebene Möglichkeit zu partiellem Schutz vor den Marktmechanismen bzw. zu deren Korrektur über solidarische Systeme auf europäischer Ebene abzusichern.

\section{Literatur}

Adamy, W., 1989: Soziale Grundrechte in der Europäischen Gemeinschaft - Zwischen leeren Versprechungen und sozialem Fortschritt', in: WSI-Mitteilungen 42, 10, 550-557.

Adamy, W., 1990: Europa für die Arbeitnehmer?, in: Böhme, H./Peressin, A. (Hrsg.), Sozialraum Europa - Die soziale Dimension des Europäischen Binnenmarktes, Frankfurt a.M., 143-162.

Agence Europe S.A, Agence internationale pour la presse (täglicher Nachrichtendienst zur europäischen Integration, Brüssel/Luxemburg).

Arbeitskreis Außenpolitik der SPD-Bundestagsfraktion, Horst Ehmke (Hrsg.), 1988: Europäischer Binnenmarkt - Europäischer Sozialraum (Zusammenfassung der Redebeiträge der Anhörung vom 2.5.1988, Fraktion der SPD im Deutschen Bundestag), Bonn.

Assicredito (associazione sindicale fra le aziende del credito) (Hrsg.), 1995: Maastricht Social Protocol: reality and perspectives, Rome: Notiziario di guirisprudenza del lavoro Supplement.

Aubry, M., 1989: Pour une Europe sociale: objectifs et méthode (extrait de: Pour une Europe sociale, rapport au ministre du Travail, de l'emploi et de la Formation professionelle, Collection des rapports officiels, septembre 1988)', Problèmes économiques 1989, 2.123, 2-5. 
Beckmann, D./John, G.,/Wathling, U., 1991: Europa '92: Grenzenloser Sozialstaat oder unsoziale Grenzenlosigkeit, in: Heine, M./Kisker, K. P./Schikora, A. (Hrsg.), Schwarzbuch EG-Binnenmarkt: Die vergessenen Kosten der Integration, Berlin, 115-133.

Bercusson, B., 1995: The Collective Labour Law of the European Union, in: European Law Journal 1, 2, 157-179.

Beretta-Bericht, 1987: Informationsbericht der Fachgruppe Sozial- und Familienfragen, Bildungswesen und Kultur des Wirtschafts- und Sozialausschusses der EG über die sozialen Aspekte des Binnenmarktes (Europäischer Sozialraum; 225/87 final vom 17. 9. 1987), Berichterstatter: Herr Beretta, Brüssel.

Betten, L./Harris, D./Jaspers, T. (Hrsg.), 1989: The Future of European Social Policy, Views and comments expressed at the conference on The Future of European Social Policy, University of Utrecht (The Netherlands), 25 and 26 April 1989, Deventer/Boston.

Beutler, B./Bieber, R./Pipkorn, J./Streil, J., 1987: Die Europäische Gemeinschaft - Rechtsordnung und Politik, 3. Aufl., Baden-Baden.

Birk, R. (Hrsg.), 1990: Die soziale Dimension des Europäischen Binnenmarktes (Referate der Tagung des Arbeitskreises Europäische Integration e.V. in Hamburg vom 19. - 21. Oktober 1989), Baden-Baden.

Böhme, H./Peressin, A., 1990: Sozialraum Europa - Die soziale Dimension des Europäischen Binnenmarktes (Sozialökonomische Schriften 3, hrsg. von B. Rürup), Frankfurt a.M./Bern/New York/ Paris.

Böttcher, W. (Hrsg.), 1990: Soziales Europa 1993 - Noch eine Illusion. Ein Arbeitsbuch, Baden-Baden.

Breit, E. (Hrsg.), 1988: Europäischer Binnenmarkt: Wirtschafts- oder Sozialraum?, Bonn.

Brok, E., 1988: Soziale Aspekte des europäischen Binnenmarkts (Europa als Auftrag 9, EVP-Fraktion im Europäischen Parlament und CDU/CSU-Fraktion im Deutschen Bundestag), Brüssel.

Bukold, S./Musiolik, S., 1990: EG-Binnenmarkt 1992: Quelle neuen Wohlstands oder trojanisches Pferd des Sozialabbaus? Chancen, Risiken und Optionen aus sozialer Perspektive, in: Strübel, $M$. (Hrsg.), Wohin treibt Europa? Der EG-Binnenmarkt und das gemeinsame europäische Haus, Marburg, 11-33.

Busch, K., 1993: Gewerkschaftliche Tarifpolitik in Europa - Handlungsbedingungen und -möglichkeiten bei unterschiedlichen europäischen Einigungsstrategien, Hans Böckler Stiftung.

Busch, K., 1994: Europäische Integration und Tarifpolitik. Lohnpolitische Konsequenzen der Wirtschafts- und Währungsunion, Köln.

Busch, K., 1997: Europäische Sozialpolitik - das Korridormodell (Vorstudie im Auftrag der HansBöckler-Stiftung), Bramsché.

Busch, K., 1998: Das Korridormodell - ein Konzept zur Weiterentwicklung der EU-Sozialpolitik, in: Internationale Politik und Gesellschaft 2, 147-156 (auch veröffentlicht in: Schmid, J./Niketta, R. (Hrsg.), Wohlfahrtsstaat - Krise und Reform im Vergleich, Marburg 1998, 273-295, und als: „The Corridor Model - a concept for further development of an EU Social Policy“, ETUI Discussion and Working Paper, Brussels 1998).

Chassard, Y., 1990: Pour une convergence des politiques des Etats membres de la Communauté dans le domaine de la protection sociale, in: Droit Social 2, 157-165.

Däubler, W. (Hrsg.), 1989a: Sozialstaat EG? Die andere Dimension des Binnenmarktes, Gütersloh.

Däubler, W., 1989b: Sozialraum Europa - Dumping oder sozialer Fortschritt, in: Steinkübler, F. (Hrsg.), Europa '92, Hamburg, 145-156.

Däubler, W., 1989c: Sozialstaat EG? Notwendigkeit und Inhalt einer Europäischen Grundrechtsakte, in: Däubler, W. (Hrsg.), Sozialstaat EG? Die andere Dimension des Binnenmarktes, Gütersloh, 35-160.

Däubler, W., 1996: Plädoyer für eine europäische Sozialpolitik, in: Ohr, R./Caesar, R. (Hrsg.), Maastricht und Maastricht II: Vision oder Abenteuer? (Schriften zur monetären Ökonomie 40, hrsg. von D. Duwendag), Baden-Baden, 147-158. 
Deubner, C. (Hrsg.), 1990: Europäische Einigung und soziale Frage - Möglichkeiten europäischer Sozialpolitik (Deutsch-französische Studien zur Industriegesellschaft 12, hrsg. von L. Kißler and R. Lasserre), Frankfurt a.M./New York.

Deutscher Gewerkschaftsbund, 1987: Für ein soziales Europa: Prioritäten der Gewerkschaften für die deutsche EG-Präsidentschaft (Beschluß des geschäftsführenden Bundesvorstandes des DGB vom 7.12.1987).

Dispersyn, M./Van der Vorst, P. u.a., 1990: La construction d'un serpent social européen, in: Revue Belge de Sécurité Sociale 12.

Europäischer Gewerkschaftsbund, 1988: Die Soziale Dimension des Binnenmarktes (Entschließung des Exekutivausschusses vom 6./7.10.1988 in Madrid).

Europäischer Gewerkschaftsbund, 1989: Europas Zukunft: Eine starke Interessenvertretung in Europas Unternehmen (Mobilisierungskampagne, Konferenz in Ostende, 16./17.10. 1989), Gruppe I: Europäischer Vergleich der Arbeitnehmervertretungen in den Unternehmen, Gruppe III: Die Rolle der Arbeitnehmervertreter im Bereich der Ausbildung, insbesondere der Weiterbildung, Brüssel.

Europäisches Gewerkschaftsinstitut, 1988: Die soziale Dimension des Binnenmarktes, Teil 2: Arbeitnehmerrrechte in den europäischen Unternehmen (Info 26), Brüssel.

Falkner, G., 1992: Soziale Sicherung und europäische Integration, in: Tálos, E. (Hrsg.), Der geforderte Wohlfahrtsstaat. Traditionen - Herausforderungen - Perspektiven, Wien, 171-193.

Falkner, G., 1993a: Sozialpartnerschaftliche Politikmuster und Europäische Integration, in: Tálos, E. (Hrsg.), Sozialpartnerschaft. Kontinuität und Wandel eines Modells, Wien, 79-102.

Falkner, G., 1993b: „Sozialdumping“ im EG-Binnenmarkt: Betrachtungen aus politikwissenschaftlicher Sicht, in: Österreichische Zeitschrift für Politikwissenschaft 22, 3, 261-277.

Falkner, G., 1996: European Works Councils and the Maastricht Social Agreement: Towards a New Policy Style?, in: Journal of European Public Policy 3, 2, 192-208.

Falkner, G., 1998: Social Europe in the 1990s: Towards a corporatist policy community, London/New York.

Falkner, G., 2000: Problemlösungsfähigkeit im europäischen Mehrebenensystem: Die soziale Dimension, in: Grande, E./Jachtenfuchs, M. (Hrsg.), Wie problemlösungsfähig ist die EU?, BadenBaden (i.E.).

Fels, G., 1989: Die Sozialcharta ökonomisch gesehen, in: Däubler, W. (Hrsg.), Sozialstaat EG? Die andere Dimension des Binnenmarktes, Gütersloh, 161-174.

Fitzpatrick, B., 1992: Community Social Law after Maastricht, in: Industrial Law Journal 21, 3, 199-213.

Franzmeyer, F., 1988: Economic, Social and Political Costs of Completing the Internal Market, in: Bieber/Dehousse/Pinder/Weiler (Hrsg.), One European Market?, Baden-Baden, 55-72.

Gerbet, P., 1983: La Construction de l'Europe, Paris.

Goetschy, J., 1994: A Further Comment on Wolfgang Streeck's ,European Social Policy after Maastricht', in: Economic and Industrial Democracy 15, 477-485.

Hörburger, H., 1990: Europas Frauen fordern mehr - Die soziale Dimension des EG-Binnenmarktes am Beispiel der spezifischen Auswirkungen auf Frauen, Marburg.

Hoskyns, C., 1986: Frauenpolitik in der Europäischen Gemeinschaft - Gleichstellungsgesetze und Frauenbewegung, in: Das Argument 159, 651-665.

Jonckheer, P./Pochet, P., 1990: De la charte sociale au programme d'action de la Communauté européenne, Brüssel.

Keller, B., 1993: Die soziale Sicht des Binnenmarktes. Zur Begründung einer euro-pessimistischen Sicht, in: Politische Vierteljahresschrift 34, 4, 588-612.

Keller, B., 1997: Europäische Arbeits- und Sozialpolitik, München/Wien.

Knigge, A., 1989: Der europäische Sozialraum: Beruhigungspille oder Chance zum Fortschritt? Stand und Möglichkeiten einer europäischen Sozialpolitik, in: Soziale Sicherheit (BRD), 1, 17-25. 
Kobler-Koch, B., 1996: Catching up with change: the transformation of governance in the European Union, in: Journal of European Public Policy 3, 3, 359-380.

Lange, P., 1993: Maastricht and the Social Protocol: Why Did They Do It?, in: Politics and Society $21,1,5-36$.

Leibfried, S., 1990: Sozialstaat Europa? Integrationsperspektiven europäischer Armutsregimes, in: Nachrichtendienst des Deutschen Vereins für öffentliche und private Fürsorge (NDV), 9/70, 295-304.

Leibfried, S., 1992: Europe's Could-Be Social State: Social Policy in European Integration after 1992, in: Adams, W. J. (Hrsg.), Singular Europe: Economy and Polity of the European Community after 1992, Michigan/USA, 97-121.

Leibfried, S./Pierson, P., 1992: Prospects for Social Europe, in: Politics \& Society 20, 3, 333-366.

Leibfried, S./Pierson, P. (Hrsg.), 1995: European Social Policy: Between Fragmentation and Integration, Washington/DC.

Lodge, J., 1989: Social Europe: Fostering a People’s Europe?, in: Lodge, J., (Hrsg.), The European Community and the Challenge of the Future, London, 303-319.

Majone, G., 1993: The European Community between Social Policy and Social Regulation, in: Journal of Common Market Studies 31, 2, 153-175.

Marin, M., 1988: La Dimension Sociale du Marché Intérieur et les Priorités de la Politique Sociale Communautaire pour 1988 (Communication de M. Marin), Brüssel.

Maydell, B. v. (Hrsg.), 1990: Soziale Rechte in der EG, Berlin.

Mayntz, R./Scharpf, F.W., 1995: Der Ansatz des akteurszentrierten Institutionalismus, in: Mayntz, R./Scharpf, F.W., (Hrsg.), Gesellschaftliche Selbstregulierung und politische Steuerung, Frankfurt a.M./New York, 39-72.

Nelhans, J., 1975: Die Freizügigkeit der Arbeitnehmer in Europa, Baden-Baden.

Observatoire Social Européen (Hrsg.), 1989: Europe: Le défi social, Brüssel.

Partikel, H., 1990: Europäische Einigung und soziale Frage - Möglichkeiten europäischer Sozialpolitik, in: Deubner, C./Kißler, L./Lasserre, R. (Hrsg.), Deutsch-französische Studien zur Industriegesellschaft, Frankfurt a.M./New York, 124-140.

Platzer, H.-W., 1996: Erosionsvehikel, Rettungsanker oder Gestaltungsfaktor? Die Auswirkungen der Europäischen Union auf den Sozialstaat, in: Internationale Politik und Gesellschaft 1, 23-35.

Pierson, P., 1996: The Path to European Integration: A Historical Institutionalist Analysis, in: Comparative Political Studies 29, 2, 123-163.

Pierson, P./Leibfried, S., 1995a: Multitiered Institutions and the Making of Social Policy, in: Leibfried, S./Pierson, P. (Hrsg.), European Social Policy: Between Fragmentation and Integration, Washington/DC, 1-40.

Pierson, P./Leibfried, S., 1995b: The Dynamics of Social Policy Integration, in: Leibfried, S./Pierson, P. (Hrsg.), European Social Policy: Between Fragmentation and Integration, Washington/DC, 432-466.

Pipkorn, J., 1986: Vorbemerkung zu Art. 117-122 EWGV und Kommentar zu Art. 117 EWGV, in: v. d. Groeben, H. et al. (Hrsg.), Handbuch des Europäischen Rechts, IA 56, Baden-Baden, 1-26 und 26-40.

Pochet, P., 1991: Social: le consensus impossible?, in: Notabene 62, 4-5.

Rhodes, M., 1995: A Regulatory Conundrum: Industrial Relations and the ,Social Dimension', in: Leibfried, S./Pierson, P. (Hrsg.), Fragmented Social Policy: The European Union's Social Dimension in Comparative Perspective, Washington/DC, 78-122.

Ross, G., 1994: On Half-Full Glasses, Europe and the Left: Comments on Wolfgang Streeck's ,European Social Policy after Maastricht', in: Economic and Industrial Democracy 15, 486-496.

Ross, G., 1995: Jacques Delors and European Integration, Cambridge.

Ross, G., 1997: The Commission As Stimulator of Interest Representation - Two Cases (paper presented at the Fifth Biennial International Conference of the European Community Studies Association USA, Seattle, May 29-June 1). 
Scharpf, F. W., 1985: Die Politikverflechtungsfalle, Europäische Integration und Deutscher Föderalismus im Vergleich, in: Politische Vierteljahresschrift 26, 4, 323-357.

Scharpf, F. W., 1994: Community and autonomy: multi-level policy-making in the European Union, in: Journal of European Public Policy 1, 2, 219-239.

Scharpf, F. W., 1995: Demokratische Politik in Europa, in: Staatswissenschaften und Staatspraxis 6, $565-591$.

Scharpf, F. W., 1996: Politische Optionen im vollendeten Binnenmarkt, in: Jachtenfuchs, M./KohlerKoch, B. (Hrsg.), Europäische Integration, Opladen, 109-140.

Scharpf, F. W., 1997a: Globalisierung als Beschränkung der Handlungsmöglichkeiten nationalstaatlicher Politik (MPIfG Discussion Paper 97/1, Max-Planck-Institut für Gesellschaftsforschung, Köln).

Scharpf, F. W., 1997b: Employment and the Welfare State: A Continental Dilemma (MPIfG Working Paper 97/7, Max-Planck-Institut für Gesellschaftsforschung, Köln).

Scharpf, F. W., 1997c: Introduction: the problem-solving capacity of multi-level governance, in: JEPP 4, 4, 520-538.

Scharpf, F. W., 1998a: Die Problemlösungsfähigkeit der Mehrebenenpolitik in Europa, Vortrag, DFG-Schwerpunktprogramm ,Regieren in der EG', Konferenz 13./14.07.1998 „Demokratisches Regieren jenseits des Staates“, Universität Mannheim, Mannheimer Zentrum für Europäische Sozialforschung (MZES).

Scharpf, F., 1998b: Balancing Positive and Negative Integration: The Regulatory Options for Europe, in: Dettke, D. (Hrsg.), The Challenge of Globalization for Germany's Social Democracy, New York/Oxford, 29-60.

Schmitter, P. C., 1981: Interest Intermediation and Regime Governability in Contemporary Western Europe and North America, in: Berger, S. (Hrsg.), Organizing Interests in Western Europe: Pluralism, Corporatism, and the Transformation of Politics, Cambridge, 287-327.

Schulte, B., 1985: Europäische Sozialpolitik - eine Zwischenbilanz, in: Sozialer Fortschritt 1/2.

Schulte, B., 1991: Die Folgen der EG-Integration für die wohlfahrtsstaatlichen Regimes, in: Zeitschrift für Sozialreform 9, 248-579.

Schulz, O., 1991: Grundsätze, Inhalte und institutionelle Verankerung im EWG-Vertrag - Überlegungen zur europäischen Sozialpolitik in der Zukunft, in: Sozialer Fortschritt 6/7, 135-140.

Schulz, O., 1996: Maastricht und die Grundlagen einer Europäischen Sozialpolitik, Köln u.a.

Steinkühler, F. (Hrsg.), 1989: Europa '92 - Industriestandort oder sozialer Lebensraum, Hamburg.

Streeck, W., 1994: European Social Policy after Maastricht: The ,Social Dialogue' and ,Subsidiarity', in: Economic and Industrial Democracy 15, 1, 151-177.

Streeck, W., 1995a: Neo-Voluntarism: A New European Social Policy Regime?, in: European Law Journal 1, 1, 31-59.

Streeck, W., 1995b: Politikverflechtung und Enscheidungslücke. Zum Verhältnis von zwischenstaatlichen Beziehungen und sozialen Interessen im europäischen Binnenmarkt, in: Bentele, K./Reissert, B./Schettkat, R. (Hrsg.), Die Reformfähigkeit von Industriegesellschaften. Fritz W. ScharpfFestschrift zu seinem 60. Geburtstag, Frankfurt a.M./New York, 101-130.

Streeck, W., 1996: Gewerkschaften zwischen Nationalstaat und Europäischer Union (MPIfG Working Paper 96/1, Max-Planck-Institut für Gesellschaftsforschung, Köln).

Streeck, W., 1997: Citizenship Under Regime Competition: The Case of the „European Works Councils“, in: European Integration online Papers 1, 5 [http://eiop.or.at/eiop/texte/1997-005a.htm].

Streeck, W., 1998: Gewerkschaften zwischen Nationalstaat und Europäischer Union, in: WSI Mitteilungen $51,1,1-14$.

Streeck, W., 1999: Competitive Solidarity: Rethinking the European Social Model (MPIfG Working Paper 1999/8), Max-Planck-Institut für Gesellschaftsforschung, Köln).

Streeck, W./Schmitter, P. C., 1994: From National Corporatism to Transnational Pluralism: Organized Interests in the Single European Market, in: Eichener, V./Voelzkow, H. (Hrsg.), Europäische Integration und verbandliche Interessenvermittlung, Marburg, 171-215 (Erstpublikation: politics and society 1991). 
Tichy, G., 1998a: Wirkungen und Herausforderungen der Globalisierung - Innovation und Technologie, in: Conturen II, 9.

Tichy, G., 1998b: Makroökonomische Beschäftigungspolitik. Beschäftigungsprobleme aus Mangel an Politikkoordination innerhalb der Europäischen Union, in: Pfusterschmid-Hartenstein, $H$. (Hrsg.), Die zerrissene Gesellschaft, Wien, 225-234.

Traxler, F./Schmitter, P. C., 1995a: The Emerging Euro-Polity and Organized Interests, in: European Journal of International Relations 1, 2, 191-218.

Traxler, F./Schmitter, P. C., 1995b: Arbeitsbeziehungen und europäische Integration, in: Mesch, M. (Hrsg.), Sozialpartnerschaft und Arbeitsbeziehungen in Europa, Wien, 231-256.

Visser, J., 1997: Learning to Play: The Europeanisation of Trade Unions (paper presented at the Fifth Biennial International Conference of the European Community Studies Association USA, Seattle, May 29-June 1).

Vogel-Polsky, E., 1989: L'Acte unique ouvre-t-il l'espace social européen?, in: Droit Social 51, 2, 177-189.

Vogel-Polsky, E., 1990: Quel futur pour L'Europe sociale après le sommet de Strasbourg?, in: Droit Social 52, 2, 219-226.

Vogel-Polsky, E., 1994: Maastricht ou la voie ètroite du social, in: Telò, M. (Hrsg.), Quelle union social européenne?, Brussels, 79-96.

Wallace, H., 1996: Die Dynamik des EU-Institutionengefüges, in: Jachtenfuchs, M./Kohler-Koch, B. (Hrs.), Europäische Integration, Opladen, 141-164.

Watson, P., 1993: Social Policy after Maastricht, in: Common Market Law Review 30, 481-513.

Weiss, M., 1992: The Significance of Maastricht for European Community Social Policy, in: The International Journal of Comparative Labour Law and Industrial Relations 8, 1, 3-15. 